Acta Crystallographica Section E

Structure Reports

Online

ISSN 1600-5368

\section{2-Amino-6-\{[(6-chloropyridin-3-yl)- methyl](ethyl)amino\}-1-methyl-5-nitro- 4-phenyl-1,4-dihydropyridine-3- carbonitrile ethanol monosolvate}

\author{
Chuan-Wen Sun, ${ }^{*}$ Yan-Xia Chen and Tian-Yan Liu \\ Department of Chemistry, College of Life and Environmental Science, Shanghai \\ Normal University, Shanghai 200234, People's Republic of China \\ Correspondence e-mail: willin112@163.com
}

Received 19 February 2012; accepted 19 March 2012

Key indicators: single-crystal X-ray study; $T=298 \mathrm{~K}$; mean $\sigma(\mathrm{C}-\mathrm{C})=0.003 \AA$; disorder in solvent or counterion; $R$ factor $=0.048 ; w R$ factor $=0.135$; data-toparameter ratio $=12.9$.

In the title compound, $\mathrm{C}_{21} \mathrm{H}_{21} \mathrm{ClN}_{6} \mathrm{O}_{2} \cdot \mathrm{C}_{2} \mathrm{H}_{6} \mathrm{O}$, a member of the insecticidal active neonicotinoid group of compounds, the 1,4dihydropyridine ring adopts a boat conformation. An intramolecular $\mathrm{C}-\mathrm{H} \cdots \mathrm{O}$ hydrogen bond occurs while the components are linked by an $\mathrm{N}-\mathrm{H} \cdots \mathrm{O}$ interaction. The crystal packing is stablized by $\mathrm{O}-\mathrm{H} \cdots \mathrm{N}$ hydrogen bonds and $\mathrm{C}-\mathrm{H}$... O interactions.

\section{Related literature}

For the synthesis, see: Zhang et al. (2010). For the insectidal activity of nitenpyram [systematic name: $(E)-N$-(6-Chloro-3pyridylmethyl)- $N$-ethyl- $N^{\prime}$-methyl-2-nitrovinylidenediamine], see: Elbert \& Nauen (2000); Jeschke \& Nauen (2008); Kashiwada (1996); Minamida et al. (1993); Shao et al. (2008); Tomizawa \& Casida (2009).<smiles>CCOCCN(Cc1ccc(Cl)nc1)C1C([N+](=O)[O-])C(c2ccccc2)C(C#N)=C(N)N1C</smiles>

\section{Experimental}

Crystal data

$\mathrm{C}_{21} \mathrm{H}_{21} \mathrm{ClN}_{6} \mathrm{O}_{2} \cdot \mathrm{C}_{2} \mathrm{H}_{6} \mathrm{O}$

$M_{r}=470.96$
Orthorhombic, $\mathrm{Pbca}$

$a=19.3334$ (19) ^

$b=12.1156(12) \AA$

$c=20.644(2) \AA$

$V=4835.5(8) \AA^{3}$

$Z=8$

Mo $K \alpha$ radiation

$\mu=0.19 \mathrm{~mm}^{-1}$

$T=298 \mathrm{~K}$

$0.16 \times 0.12 \times 0.10 \mathrm{~mm}$

Data collection

Bruker SMART CCD area-detector diffractometer

Absorption correction: multi-scan (SADABS: Bruker, 2001)

$T_{\min }=0.970, T_{\max }=0.981$

Refinement

$R\left[F^{2}>2 \sigma\left(F^{2}\right)\right]=0.048$

$w R\left(F^{2}\right)=0.135$

$S=1.05$

4267 reflections

330 parameters

44471 measured reflections 4267 independent reflections 3044 reflections with $I>2 \sigma(I)$ $R_{\text {int }}=0.045$

Table 1

Hydrogen-bond geometry $\left(\AA,^{\circ}\right)$.

\begin{tabular}{lllll}
\hline$D-\mathrm{H} \cdots A$ & $D-\mathrm{H}$ & $\mathrm{H} \cdots A$ & $D \cdots A$ & $D-\mathrm{H} \cdots A$ \\
\hline $\mathrm{O} 3-\mathrm{H} 3 A \cdots \mathrm{N} 1^{\mathrm{i}}$ & 0.82 & 1.97 & $2.785(13)$ & 179 \\
$\mathrm{~N} 4-\mathrm{H} 4 B \cdots \mathrm{O} 3$ & 0.86 & 2.26 & $2.902(11)$ & 132 \\
$\mathrm{C} 6-\mathrm{H} 6 A \cdots \mathrm{O} 2$ & 0.97 & 2.13 & $2.783(3)$ & 124 \\
$\mathrm{C} 7-\mathrm{H} 7 B \cdots \mathrm{O} 2^{\mathrm{ii}}$ & 0.97 & 2.57 & $3.378(3)$ & 141 \\
\hline
\end{tabular}

Symmetry codes: (i) $x,-y+\frac{1}{2}, z-\frac{1}{2}$; (ii) $-x+\frac{1}{2}, y-\frac{1}{2}, z$.

Data collection: SMART (Bruker, 2001); cell refinement: SAINTPlus (Bruker, 2001); data reduction: SAINT-Plus; program(s) used to solve structure: SHELXS97 (Sheldrick, 2008); program(s) used to refine structure: SHELXL97 (Sheldrick, 2008); molecular graphics: PLATON (Spek, 2009); software used to prepare material for publication: PLATON.

This work was supported financially by the National Natural Science Foundation of China (21042010, 21102092 and 30870560).

Supplementary data and figures for this paper are available from the IUCr electronic archives (Reference: GG2077).

\section{References}

Bruker (2001). SADABS, SMART and SAINT-Plus. Bruker AXS Inc., Madison, Wisconsin, USA.

Elbert, A. \& Nauen, R. (2000). Pest Manage. Sci. 56, 60-64.

Jeschke, P. \& Nauen, R. (2008). Pest Manage. Sci. 64, 1084-1098.

Kashiwada, Y. (1996). Agrochem. Jpn, 68, 18-19.

Minamida, I., Iwanaga, K. \& Tabuchi, T. (1993). J. Pestic. Sci. 18, 31-40.

Shao, X. S., Zhang, W. W., Peng, Y. Q., Li, Z., Tian, Z. Z. \& Qian, X. H. (2008). Bioorg. Med. Chem. Lett. 18, 6513-6516.

Sheldrick, G. M. (2008). Acta Cryst. A64, 112-122.

Spek, A. L. (2009). Acta Cryst. D65, 148-155.

Tomizawa, M. \& Casida, J. E. (2009). Acc. Chem. Res. 42, 260-269.

Zhang, W. W., Yang, X. B., Chen, W. D., Xu, X. Y., Li, L., Zhai, H. B. \& Li, Z. (2010). J. Agric. Food Chem. 58, 2741-2745. 


\section{supporting information}

Acta Cryst. (2012). E68, o1146 [https://doi.org/10.1107/S1600536812011750]

\section{2-Amino-6-\{[(6-chloropyridin-3-yl) methyl] (ethyl)amino\}-1-methyl-5-nitro-4- phenyl-1,4-dihydropyridine-3-carbonitrile ethanol monosolvate}

\section{Chuan-Wen Sun, Yan-Xia Chen and Tian-Yan Liu}

\section{S1. Comment}

Neonicotinoid insecticides (NNSs), which act agonistically on the insect nicotinic acetylcholine receptors (nAChRs), are gaining widespread use as a way to control pests, because of their high potency and low mammalian toxicity. As part of the chloronicotinyl subclass, nitenpyram, which was brought to the market two decades ago, also showed higher selectivity and better systemic properties against mammals, birds, aquatic life than insects, due to the differential binding affinities with the nAChR receptors of their neurosystem. (Jeschke \& Nauen, 2008; Tomizawa \& Casida, 2009; Minamida et al., 1993; Kashiwada, 1996; Shao et al., 2008; Elbert \& Nauen, 2000). In this report, the title compound (Scheme I) was synthesized and characterized by X-ray diffraction.

In the title structure, $\mathrm{C}_{21} \mathrm{H}_{21} \mathrm{ClN}_{6} \mathrm{O}_{2} \mathrm{C}_{2} \mathrm{H}_{6} \mathrm{O}$, (I), there is a cis-2-Amino-6-[N-(6-chloro-3-pyridinylmethyl)-N-ethyl]amino-3-cyano-1-methyl-5- nitro-4-phenyl-1,4-dihydropyridine molecule and a ethanol molecule in the asymmetric unit (Fig. 1). The 1,4-dihydropyridine ring adopts a sofa (boat) conformation. As compared with the trans configuration of nitro in the crystal structure of nitenpyram, the nitro group in the title compound is in the cis configuration as anticipated. Interestingly, the $\mathrm{C}-\mathrm{C}$ and $\mathrm{C}-\mathrm{N}$ bond length data (C9-N3 1.389 (2) $\AA, \mathrm{N} 3-\mathrm{C} 101.402$ (3) $\AA, \mathrm{C} 11-\mathrm{C} 121.510$ (3) $\AA$ and C12-C13 1.519 (3) $\AA)$ in the structure of (I) are shorter than the standard $\mathrm{C}-\mathrm{C}(1.54 \AA)$ and $\mathrm{C}-\mathrm{N}(1.47 \AA)$. On the contrary, the $\mathrm{C}=\mathrm{C}$ bond length data $(\mathrm{C} 9=\mathrm{C} 131.384$ (3) $\AA$ and $\mathrm{C} 10=\mathrm{C} 111.348$ (3) $\AA$ ) are longer than the standard $\mathrm{C}=\mathrm{C}$ bond (1.34 $\AA$ ). This shows that there is a homo-conjugation effect on the 1,4-dihydropyridine scaffold (Fig. 1).

The crystal packing is stablized by $\mathrm{O}-\mathrm{H} \cdots \mathrm{N}, \mathrm{N}-\mathrm{H} \cdots \mathrm{O}$ and $\mathrm{C}-\mathrm{H} \cdots \mathrm{O}$ hydrogen bonds (Fig. 2). Analysis shows that no intermolecular $\mathrm{p} \cdots \pi$ or $\mathrm{C}-\mathrm{H} \cdots \pi$ interactions exist in the crystal structure.

\section{S2. Experimental}

The title compound was prepared by the literature method (Zhang et al., 2010) and it was obtained using volatilization of petroleum ether and ethanol solution at room temperature, giving yellow crystals (yield 83.7\%). ${ }^{1} \mathrm{H} \mathrm{NMR}\left(\mathrm{CDCl}_{3}, 400\right.$ Hz): 8.08 (d, $J=12.4 \mathrm{~Hz}, 1 H, \mathrm{Py}-\mathrm{H}), 7.34$ (d, $J=8.9 \mathrm{~Hz}, 1 H, \mathrm{Py}-\mathrm{H}), 7.24$ (s,3H,Ph-H), 7.08 (m, $J=7.8 \mathrm{~Hz}, 1 H, \mathrm{Py}$ -H), 7.05-6.95 (m, 2H, Ph-H), $5.06(\mathrm{~s}, 1 H, \mathrm{CH}), 4.79\left(\mathrm{~s}, 2 H, \mathrm{NH}_{2}\right), 4.33(\mathrm{~d}, J=14.8 \mathrm{~Hz}, 1 H), 4.06(\mathrm{~m}, J=14.6 \mathrm{~Hz}$, $1 H), 3.35-3.20(\mathrm{~m}, 1 H), 3.17$ (s, $\left.3 H, \mathrm{NCH}_{3}\right), 3.10(\mathrm{~d}, J=7.3 \mathrm{~Hz}, 1 H), 1.33-1.21\left(\mathrm{~m}, 3 H, \mathrm{NCH}_{2} \mathrm{CH}_{3}\right)$. IR( $\left(\mathrm{KBr}, \mathrm{cm}^{-1}\right) 2974$ $\left(\mathrm{CH}_{3}\right), 3327,3197\left(\mathrm{NH}_{2}\right), 2184(\mathrm{CN}), 1457,1409\left(\mathrm{NO}_{2}\right), 1648,1614,1557$ (benzene).Anal. calcd. for $\mathrm{C}_{23} \mathrm{H}_{21} \mathrm{ClN}_{6} \mathrm{O}_{2} \mathrm{C}$ 59.36, H 4.98, N 19.78\% found, C 59.38, H 4.97, N 19.76\%.

\section{S3. Refinement}

During the refinement, the ethanol molecule was disordered over two sites. These $\mathrm{C}-\mathrm{C}$ and $\mathrm{C}-\mathrm{O}$ distances were refined with the restraints of $\mathrm{C}-\mathrm{C}=1.51(1) \AA$ and $\mathrm{C}-\mathrm{O}=1.38(1) \AA$ by using the DFIX command. The final occupancies for the major and minor components were 0.57 (1):0.43 (1), respectively. In (I), $\mathrm{H}$ atoms bonded to $\mathrm{C}$ and $\mathrm{N}$ atoms were located 
at their ideal positions and subsequently treated as riding modes with $\mathrm{C}-\mathrm{H}$ distances of $0.93 \AA$ (aromatic), $0.97 \AA$ (methylene) $0.98 \AA$ (methine) $0.86 \AA$ (amine) and $0.96 \AA$ (methyl) with $U_{\text {iso }}(\mathrm{H})=1.2 U_{\text {eq }}$ (aromatic, methylene, methine $\mathrm{C}$ or $\mathrm{N}$ ) or $1.5 U_{\text {eq }}$ (methyl C). $\mathrm{H}$ atoms bonded to ethanol $\mathrm{O}$ atoms were located at its ideal position $(\mathrm{O}-\mathrm{H}=0.82 \AA)$ and refined with the constraint of the $U_{\text {iso }}(\mathrm{H})=1.5 U_{\text {eq }}(\mathrm{O})$.

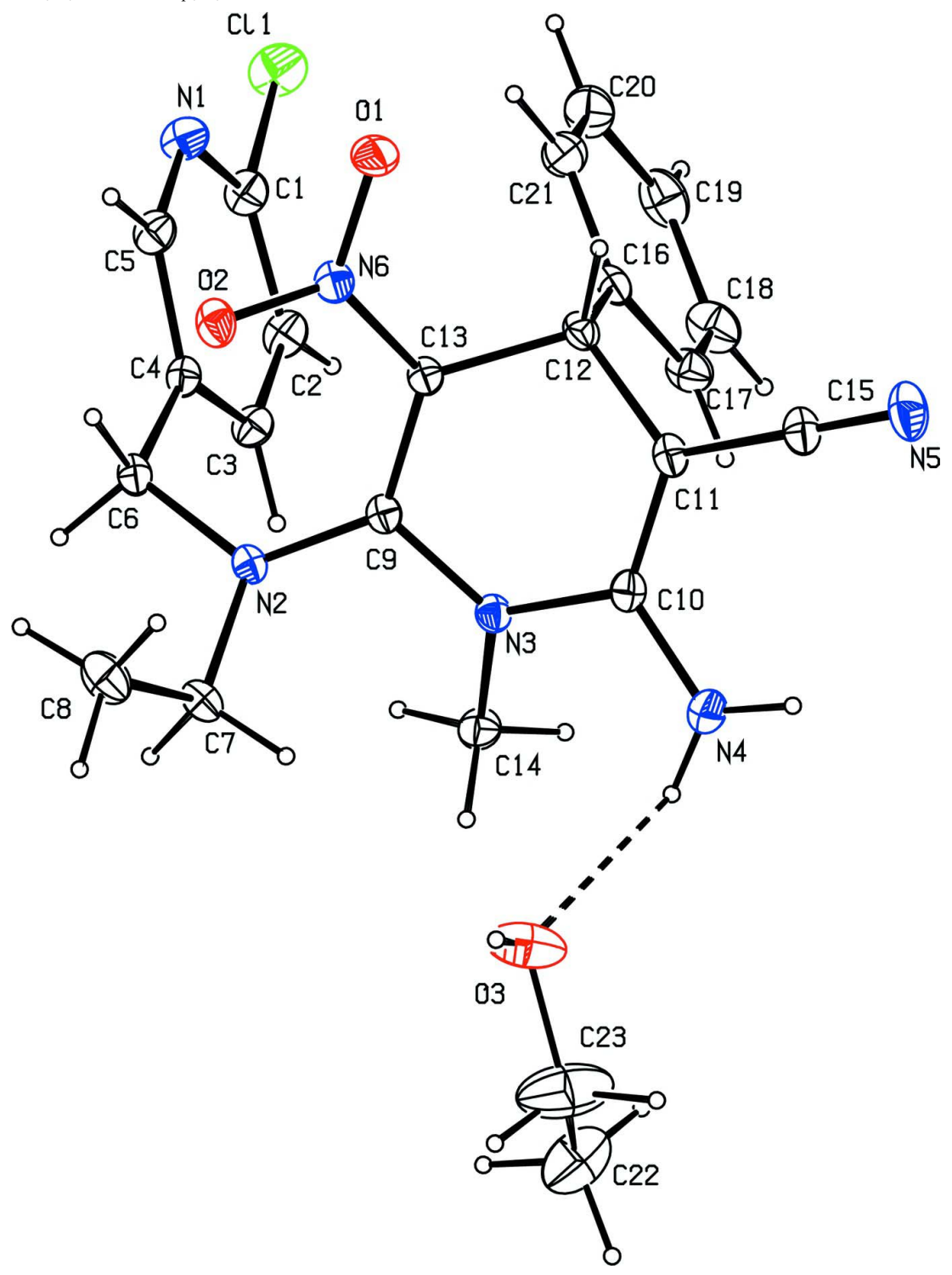

Figure 1

The molecular structure of (I) shown with 30\% displacement ellipsoids. For clarity, the minor component of the disordered ethanol molecule is omitted. Hydrogen bonding is shown as dashed lines. 


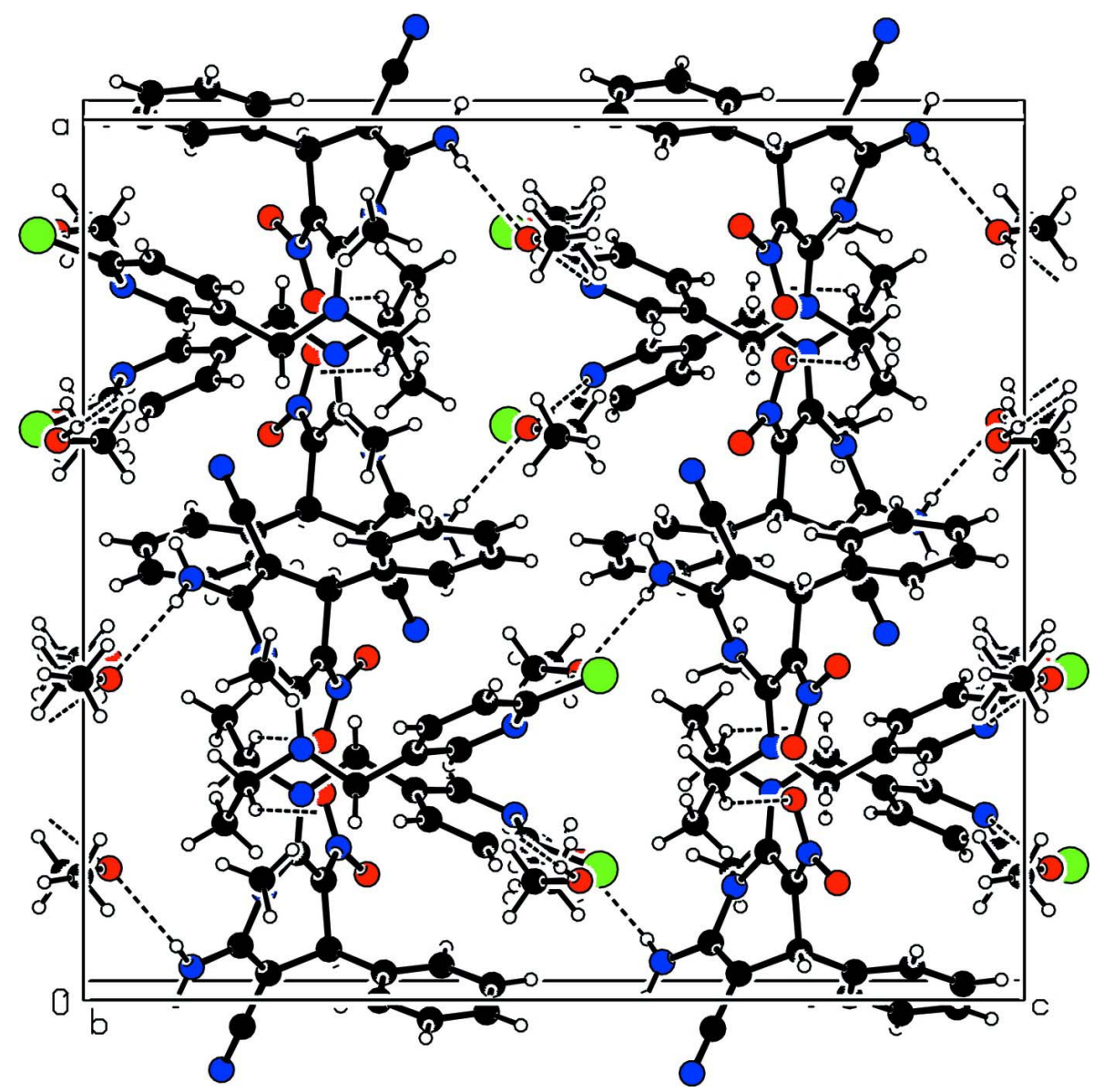

Figure 2

Part of the crystal packing in the title compound (I). Hydrogen bonds are shown as dashed lines.

2-Amino-6-\{[(6-chloropyridin-3-yl)methyl] (ethyl)amino\}-1-methyl- 5-nitro-4-phenyl-1,4-dihydropyridine-3carbonitrile ethanol monosolvate

Crystal data

$\mathrm{C}_{21} \mathrm{H}_{21} \mathrm{ClN}_{6} \mathrm{O}_{2} \cdot \mathrm{C}_{2} \mathrm{H}_{6} \mathrm{O}$

$M_{r}=470.96$

Orthorhombic, $\mathrm{Pbca}$

Hall symbol: -P 2ac $2 \mathrm{ab}$

$a=19.3334$ (19) $\AA$

$b=12.1156(12) \AA$

$c=20.644(2) \AA$

$V=4835.5(8) \AA^{3}$

$Z=8$

\section{Data collection}

Bruker SMART CCD area-detector diffractometer

Radiation source: fine-focus sealed tube Graphite monochromator phi and $\omega$ scans
$F(000)=1984$

$D_{\mathrm{x}}=1.294 \mathrm{Mg} \mathrm{m}^{-3}$

Mo $K \alpha$ radiation, $\lambda=0.71073 \AA$

Cell parameters from 5913 reflections

$\theta=2.2-20.1^{\circ}$

$\mu=0.19 \mathrm{~mm}^{-1}$

$T=298 \mathrm{~K}$

Block, yellow

$0.16 \times 0.12 \times 0.10 \mathrm{~mm}$

Absorption correction: multi-scan

(SADABS: Bruker, 2001)

$T_{\min }=0.970, T_{\max }=0.981$

44471 measured reflections

4267 independent reflections

3044 reflections with $I>2 \sigma(I)$ 


$$
\begin{aligned}
& R_{\text {int }}=0.045 \\
& \theta_{\text {max }}=25.0^{\circ}, \theta_{\text {min }}=2.0^{\circ} \\
& h=-23 \rightarrow 23
\end{aligned}
$$

\section{Refinement}

Refinement on $F^{2}$

Least-squares matrix: full

$R\left[F^{2}>2 \sigma\left(F^{2}\right)\right]=0.048$

$w R\left(F^{2}\right)=0.135$

$S=1.05$

4267 reflections

330 parameters

4 restraints

Primary atom site location: structure-invariant direct methods $k=-14 \rightarrow 14$

$l=-24 \rightarrow 24$

Secondary atom site location: difference Fourier map

Hydrogen site location: inferred from neighbouring sites

$\mathrm{H}$-atom parameters constrained

$w=1 /\left[\sigma^{2}\left(F_{\mathrm{o}}^{2}\right)+(0.0687 P)^{2}+0.8771 P\right]$ where $P=\left(F_{\mathrm{o}}^{2}+2 F_{\mathrm{c}}^{2}\right) / 3$

$(\Delta / \sigma)_{\max }<0.001$

$\Delta \rho_{\max }=0.21 \mathrm{e} \AA^{-3}$

$\Delta \rho_{\min }=-0.20$ e $\AA^{-3}$

Special details

Geometry. All esds (except the esd in the dihedral angle between two 1.s. planes) are estimated using the full covariance matrix. The cell esds are taken into account individually in the estimation of esds in distances, angles and torsion angles; correlations between esds in cell parameters are only used when they are defined by crystal symmetry. An approximate (isotropic) treatment of cell esds is used for estimating esds involving l.s. planes.

Refinement. Refinement of $\mathrm{F}^{2}$ against ALL reflections. The weighted R-factor $\mathrm{wR}$ and goodness of fit $\mathrm{S}$ are based on $\mathrm{F}^{2}$, conventional R-factors $R$ are based on $F$, with $F$ set to zero for negative $F^{2}$. The threshold expression of $F^{2}>2 \operatorname{sigma}\left(\mathrm{F}^{2}\right)$ is used only for calculating R-factors(gt) etc. and is not relevant to the choice of reflections for refinement. R-factors based

\begin{tabular}{|c|c|c|c|c|c|}
\hline & $x$ & $y$ & $z$ & $U_{\text {iso }} * / U_{\text {eq }}$ & Occ. $(<1)$ \\
\hline $\mathrm{C} 1$ & $0.16697(13)$ & $0.1575(2)$ & $0.97037(12)$ & $0.0672(6)$ & \\
\hline $\mathrm{C} 2$ & $0.16346(13)$ & $0.0715(2)$ & $0.92705(12)$ & $0.0677(6)$ & \\
\hline $\mathrm{H} 2$ & 0.1423 & 0.0051 & 0.9379 & $0.081^{*}$ & \\
\hline $\mathrm{C} 3$ & $0.19239(11)$ & $0.08776(18)$ & $0.86736(11)$ & $0.0594(6)$ & \\
\hline H3 & 0.1913 & 0.0314 & 0.8368 & $0.071^{*}$ & \\
\hline $\mathrm{C} 4$ & $0.22332(10)$ & $0.18758(16)$ & $0.85211(10)$ & $0.0509(5)$ & \\
\hline $\mathrm{C} 5$ & $0.22151(11)$ & $0.26760(19)$ & $0.89890(12)$ & $0.0636(6)$ & \\
\hline H5 & 0.2408 & 0.3359 & 0.8890 & $0.076^{*}$ & \\
\hline C6 & $0.26080(10)$ & $0.20673(18)$ & $0.78962(10)$ & $0.0538(5)$ & \\
\hline H6A & 0.2766 & 0.2827 & 0.7884 & $0.065 *$ & \\
\hline H6B & 0.3014 & 0.1596 & 0.7884 & $0.065 *$ & \\
\hline $\mathrm{C} 7$ & $0.25784(11)$ & $0.15403(18)$ & $0.67342(11)$ & $0.0608(6)$ & \\
\hline H7A & 0.2256 & 0.1348 & 0.6392 & $0.073 *$ & \\
\hline H7B & 0.2857 & 0.0893 & 0.6828 & $0.073 *$ & \\
\hline $\mathrm{C} 8$ & $0.30456(13)$ & 0.2463 & $0.65010(14)$ & $0.0890(8)$ & \\
\hline H8A & 0.2773 & 0.3106 & 0.6409 & $0.133^{*}$ & \\
\hline H8B & 0.3282 & 0.2233 & 0.6115 & $0.133^{*}$ & \\
\hline $\mathrm{H} 8 \mathrm{C}$ & 0.3379 & 0.2634 & 0.6831 & $0.133^{*}$ & \\
\hline C9 & $0.15149(10)$ & $0.21488(15)$ & $0.72709(10)$ & $0.0457(5)$ & \\
\hline $\mathrm{C} 10$ & $0.04705(11)$ & $0.17912(18)$ & $0.66673(10)$ & $0.0540(5)$ & \\
\hline C11 & $0.01366(10)$ & $0.26259(18)$ & $0.69650(11)$ & $0.0555(5)$ & \\
\hline $\mathrm{C} 12$ & $0.04016(10)$ & $0.30390(16)$ & $0.76091(10)$ & $0.0515(5)$ & \\
\hline
\end{tabular}
on $\mathrm{F}^{2}$ are statistically about twice as large as those based on F, and R- factors based on ALL data will be even larger.

Fractional atomic coordinates and isotropic or equivalent isotropic displacement parameters $\left(\AA^{2}\right)$ 


\begin{tabular}{|c|c|c|c|c|c|}
\hline H12 & 0.0249 & 0.3805 & 0.7660 & $0.062 *$ & \\
\hline $\mathrm{C} 13$ & $0.11850(9)$ & $0.30414(15)$ & $0.75527(10)$ & $0.0473(5)$ & \\
\hline $\mathrm{C} 14$ & $0.12560(12)$ & 0.02347 (17) & $0.68983(12)$ & $0.0629(6)$ & \\
\hline $\mathrm{H} 14 \mathrm{~A}$ & 0.1431 & 0.0043 & 0.6478 & $0.094 *$ & \\
\hline H14B & 0.0841 & -0.0175 & 0.6983 & $0.094^{*}$ & \\
\hline $\mathrm{H} 14 \mathrm{C}$ & 0.1596 & 0.0060 & 0.7221 & $0.094^{*}$ & \\
\hline $\mathrm{C} 15$ & $-0.04783(12)$ & $0.3063(2)$ & $0.66890(13)$ & $0.0663(6)$ & \\
\hline $\mathrm{C} 16$ & $0.01488(10)$ & $0.23895(18)$ & $0.81946(11)$ & $0.0551(5)$ & \\
\hline $\mathrm{C} 17$ & $-0.01453(13)$ & $0.1358(2)$ & $0.81419(14)$ & $0.0727(7)$ & \\
\hline H17 & -0.0206 & 0.1046 & 0.7734 & $0.087^{*}$ & \\
\hline $\mathrm{C} 18$ & $-0.03510(15)$ & $0.0781(2)$ & $0.86844(16)$ & $0.0910(9)$ & \\
\hline H18 & -0.0544 & 0.0082 & 0.8638 & $0.109^{*}$ & \\
\hline C19 & $-0.02752(15)$ & $0.1221(3)$ & $0.92841(17)$ & $0.0951(9)$ & \\
\hline H19 & -0.0416 & 0.0830 & 0.9649 & $0.114^{*}$ & \\
\hline $\mathrm{C} 20$ & $0.00120(15)$ & $0.2249(3)$ & $0.93454(15)$ & $0.0975(10)$ & \\
\hline $\mathrm{H} 20$ & 0.0067 & 0.2558 & 0.9755 & $0.117 *$ & \\
\hline C21 & $0.02208(13)$ & $0.2832(2)$ & $0.88048(13)$ & $0.0784(7)$ & \\
\hline $\mathrm{H} 21$ & 0.0412 & 0.3531 & 0.8854 & $0.094 *$ & \\
\hline $\mathrm{Cl1}$ & $0.13433(5)$ & $0.14060(7)$ & $1.04868(3)$ & $0.1000(3)$ & \\
\hline N1 & $0.19380(11)$ & $0.25445(17)$ & $0.95814(10)$ & $0.0710(6)$ & \\
\hline N2 & $0.21881(8)$ & $0.18511(13)$ & $0.73179(8)$ & $0.0486(4)$ & \\
\hline N3 & $0.11034(8)$ & $0.14244(13)$ & $0.69193(8)$ & $0.0491(4)$ & \\
\hline N4 & $0.02386(10)$ & $0.12143(17)$ & $0.61498(10)$ & $0.0750(6)$ & \\
\hline $\mathrm{H} 4 \mathrm{~A}$ & -0.0156 & 0.1370 & 0.5981 & $0.090 *$ & \\
\hline H4B & 0.0486 & 0.0692 & 0.5989 & $0.090^{*}$ & \\
\hline N5 & $-0.09771(11)$ & $0.34154(19)$ & $0.64678(13)$ & $0.0927(7)$ & \\
\hline N6 & $0.15343(9)$ & $0.40156(14)$ & $0.77108(9)$ & $0.0568(5)$ & \\
\hline $\mathrm{O} 1$ & $0.12139(9)$ & $0.47446(13)$ & $0.80126(9)$ & $0.0812(5)$ & \\
\hline $\mathrm{O} 2$ & $0.21474(8)$ & $0.41614(12)$ & $0.75479(9)$ & $0.0703(5)$ & \\
\hline $\mathrm{C} 22$ & $0.1322(6)$ & $-0.1213(7)$ & 0.4967 (5) & 0.147 (4) & 0.57 \\
\hline $\mathrm{H} 22 \mathrm{~A}$ & 0.1746 & -0.1413 & 0.5178 & $0.220 *$ & 0.57 \\
\hline H22B & 0.1263 & -0.1656 & 0.4585 & $0.220^{*}$ & 0.57 \\
\hline $\mathrm{H} 22 \mathrm{C}$ & 0.0941 & -0.1334 & 0.5256 & $0.220 *$ & 0.57 \\
\hline C23 & $0.1345(8)$ & $-0.0106(7)$ & $0.4793(5)$ & $0.194(6)$ & 0.57 \\
\hline $\mathrm{H} 23 \mathrm{~A}$ & 0.0942 & 0.0043 & 0.4528 & $0.233^{*}$ & 0.57 \\
\hline $\mathrm{H} 23 \mathrm{~B}$ & 0.1747 & -0.0004 & 0.4519 & $0.233^{*}$ & 0.57 \\
\hline $\mathrm{O} 3$ & $0.1370(7)$ & $0.0705(9)$ & $0.5276(6)$ & $0.145(5)$ & 0.57 \\
\hline $\mathrm{H} 3 \mathrm{~A}$ & 0.1532 & 0.1222 & 0.5069 & $0.218^{*}$ & 0.57 \\
\hline $\mathrm{C} 22^{\prime}$ & $0.1377(5)$ & $-0.0766(13)$ & $0.4602(5)$ & $0.113(3)$ & 0.43 \\
\hline $\mathrm{H} 22 \mathrm{D}$ & 0.0896 & -0.0859 & 0.4701 & $0.169^{*}$ & 0.43 \\
\hline $\mathrm{H} 22 \mathrm{E}$ & 0.1581 & -0.1473 & 0.4513 & $0.169^{*}$ & 0.43 \\
\hline $\mathrm{H} 22 \mathrm{~F}$ & 0.1425 & -0.0298 & 0.4229 & $0.169 *$ & 0.43 \\
\hline C23' & $0.1723(4)$ & $-0.0269(7)$ & $0.5146(4)$ & $0.094(2)$ & 0.43 \\
\hline $\mathrm{H} 23 \mathrm{C}$ & 0.1732 & -0.0788 & 0.5503 & $0.113 *$ & 0.43 \\
\hline $\mathrm{H} 23 \mathrm{D}$ & 0.2198 & -0.0105 & 0.5028 & $0.113^{*}$ & 0.43 \\
\hline O3' & $0.1403(5)$ & $0.0683(7)$ & $0.5341(6)$ & 0.085 & 0.43 \\
\hline H3B & 0.1193 & 0.0955 & 0.5035 & $0.127^{*}$ & 0.43 \\
\hline
\end{tabular}


Atomic displacement parameters $\left(\AA^{2}\right)$

\begin{tabular}{|c|c|c|c|c|c|c|}
\hline & $U^{11}$ & $U^{22}$ & $U^{33}$ & $U^{12}$ & $U^{13}$ & $U^{23}$ \\
\hline $\mathrm{C} 1$ & $0.0703(15)$ & 0.0707 (16) & 0.0606 (14) & $-0.0110(12)$ & $0.0025(12)$ & $0.0082(12)$ \\
\hline $\mathrm{C} 2$ & $0.0759(15)$ & $0.0581(14)$ & $0.0690(16)$ & $-0.0160(12)$ & $-0.0023(13)$ & $0.0120(12)$ \\
\hline $\mathrm{C} 3$ & 0.0652 (14) & $0.0515(12)$ & $0.0616(14)$ & $-0.0049(10)$ & $-0.0046(11)$ & $0.0034(11)$ \\
\hline $\mathrm{C} 4$ & $0.0448(11)$ & $0.0496(12)$ & $0.0582(13)$ & $-0.0018(9)$ & $-0.0067(9)$ & $0.0043(10)$ \\
\hline $\mathrm{C} 5$ & $0.0669(14)$ & $0.0556(13)$ & $0.0683(16)$ & $-0.0134(11)$ & $0.0021(12)$ & $0.0053(12)$ \\
\hline C6 & $0.0416(11)$ & $0.0535(12)$ & $0.0665(14)$ & $-0.0009(9)$ & $-0.0016(10)$ & $0.0037(10)$ \\
\hline $\mathrm{C} 7$ & $0.0543(12)$ & $0.0631(14)$ & $0.0651(14)$ & $0.0088(10)$ & $0.0134(11)$ & $0.0066(11)$ \\
\hline $\mathrm{C} 8$ & $0.0765(17)$ & 0.0963 (19) & $0.094(2)$ & $-0.0013(15)$ & $0.0331(15)$ & $0.0180(16)$ \\
\hline C9 & $0.0440(11)$ & $0.0428(11)$ & $0.0505(11)$ & $-0.0034(9)$ & $0.0030(9)$ & $0.0091(9)$ \\
\hline $\mathrm{C} 10$ & $0.0472(11)$ & $0.0586(13)$ & $0.0563(13)$ & $-0.0054(10)$ & $-0.0024(10)$ & $0.0052(11)$ \\
\hline C11 & $0.0430(11)$ & $0.0554(12)$ & $0.0682(14)$ & $0.0019(10)$ & $-0.0014(10)$ & $0.0085(11)$ \\
\hline C12 & $0.0435(11)$ & $0.0430(11)$ & $0.0682(14)$ & $0.0065(9)$ & $0.0034(10)$ & $0.0016(10)$ \\
\hline $\mathrm{C} 13$ & $0.0448(11)$ & $0.0389(10)$ & $0.0584(12)$ & $-0.0016(9)$ & $0.0009(9)$ & $0.0053(9)$ \\
\hline $\mathrm{C} 14$ & $0.0681(14)$ & $0.0443(12)$ & $0.0764(16)$ & $-0.0008(10)$ & $-0.0046(12)$ & $-0.0002(11)$ \\
\hline $\mathrm{C} 15$ & $0.0502(13)$ & $0.0630(14)$ & $0.0856(17)$ & $-0.0020(11)$ & $-0.0027(12)$ & $0.0091(13)$ \\
\hline $\mathrm{C} 16$ & $0.0394(10)$ & $0.0570(13)$ & $0.0690(14)$ & $0.0056(10)$ & $0.0088(10)$ & $0.0011(11)$ \\
\hline $\mathrm{C} 17$ & $0.0740(15)$ & $0.0651(15)$ & $0.0790(17)$ & $-0.0060(13)$ & $0.0211(13)$ & 0.0025 (13) \\
\hline $\mathrm{C} 18$ & $0.094(2)$ & 0.0777 (18) & $0.102(2)$ & $-0.0085(15)$ & $0.0348(18)$ & $0.0158(17)$ \\
\hline C19 & 0.0780 (19) & $0.118(3)$ & $0.090(2)$ & $0.0036(18)$ & $0.0234(16)$ & $0.033(2)$ \\
\hline $\mathrm{C} 20$ & $0.087(2)$ & $0.141(3)$ & $0.0646(18)$ & $-0.006(2)$ & $0.0106(15)$ & $0.0001(19)$ \\
\hline $\mathrm{C} 21$ & $0.0729(16)$ & 0.0889 (19) & $0.0735(18)$ & $-0.0101(14)$ & $0.0092(14)$ & $-0.0073(15)$ \\
\hline $\mathrm{Cl1}$ & $0.1254(7)$ & $0.1083(6)$ & $0.0663(5)$ & $-0.0273(5)$ & $0.0183(4)$ & $0.0100(4)$ \\
\hline N1 & $0.0846(14)$ & $0.0667(13)$ & $0.0618(13)$ & $-0.0142(11)$ & $0.0057(10)$ & $-0.0010(10)$ \\
\hline N2 & $0.0411(9)$ & $0.0498(9)$ & $0.0550(10)$ & $0.0011(7)$ & $0.0036(8)$ & $0.0046(8)$ \\
\hline N3 & $0.0455(9)$ & $0.0430(9)$ & $0.0588(10)$ & $0.0013(7)$ & $-0.0006(8)$ & $0.0022(8)$ \\
\hline N4 & $0.0625(12)$ & $0.0837(14)$ & $0.0787(14)$ & $0.0041(10)$ & $-0.0180(10)$ & $-0.0155(12)$ \\
\hline N5 & $0.0568(13)$ & $0.0909(16)$ & $0.130(2)$ & $0.0106(11)$ & $-0.0179(13)$ & $0.0186(15)$ \\
\hline N6 & $0.0557(11)$ & $0.0431(10)$ & $0.0715(12)$ & $-0.0019(9)$ & 0.0003 (9) & 0.0057 (9) \\
\hline O1 & $0.0788(11)$ & $0.0533(10)$ & 0.1115 (14) & $-0.0030(9)$ & $0.0099(10)$ & $-0.0224(10)$ \\
\hline $\mathrm{O} 2$ & $0.0534(9)$ & $0.0533(9)$ & 0.1042 (13) & $-0.0117(7)$ & $0.0058(9)$ & $0.0071(9)$ \\
\hline $\mathrm{C} 22$ & $0.203(10)$ & $0.110(7)$ & $0.128(8)$ & $-0.023(6)$ & $-0.051(7)$ & $0.017(5)$ \\
\hline $\mathrm{C} 23$ & 0.363 (19) & $0.097(7)$ & $0.122(8)$ & $0.046(9)$ & $-0.026(10)$ & $-0.009(6)$ \\
\hline $\mathrm{O} 3$ & $0.219(11)$ & $0.122(8)$ & $0.095(6)$ & $0.048(7)$ & $0.036(6)$ & $-0.014(6)$ \\
\hline $\mathrm{C} 22^{\prime}$ & $0.099(6)$ & $0.134(10)$ & $0.105(8)$ & $-0.003(7)$ & $0.013(6)$ & $-0.021(7)$ \\
\hline C23' & $0.099(5)$ & $0.105(7)$ & $0.077(5)$ & $0.040(5)$ & -0.010 & $0.013(5)$ \\
\hline $\mathrm{O}^{\prime}$ & $0.105(6)$ & $0.055(5)$ & $0.093(7)$ & $-0.001(5)$ & $0.037(5)$ & $0.016(5)$ \\
\hline
\end{tabular}

Geometric parameters $\left(\AA,{ }^{\circ}\right)$

\begin{tabular}{llll}
\hline $\mathrm{C} 1-\mathrm{N} 1$ & $1.309(3)$ & $\mathrm{C} 14-\mathrm{H} 14 \mathrm{~B}$ & 0.9600 \\
$\mathrm{C} 1-\mathrm{C} 2$ & $1.374(3)$ & $\mathrm{C} 14-\mathrm{H} 14 \mathrm{C}$ & 0.9600 \\
$\mathrm{C} 1-\mathrm{C} 11$ & $1.747(3)$ & $\mathrm{C} 15-\mathrm{N} 5$ & $1.149(3)$ \\
$\mathrm{C} 2-\mathrm{C} 3$ & $1.368(3)$ & $\mathrm{C} 16-\mathrm{C} 21$ & $1.376(3)$ \\
$\mathrm{C} 2-\mathrm{H} 2$ & 0.9300 & $\mathrm{C} 16-\mathrm{C} 17$ & $1.377(3)$ \\
$\mathrm{C} 3-\mathrm{C} 4$ & $1.385(3)$ & $\mathrm{C} 17-\mathrm{C} 18$ & $1.379(4)$
\end{tabular}




\begin{tabular}{|c|c|c|c|}
\hline $\mathrm{C} 3-\mathrm{H} 3$ & 0.9300 & C17-H17 & 0.9300 \\
\hline $\mathrm{C} 4-\mathrm{C} 5$ & $1.369(3)$ & $\mathrm{C} 18-\mathrm{C} 19$ & $1.356(4)$ \\
\hline $\mathrm{C} 4-\mathrm{C} 6$ & $1.498(3)$ & $\mathrm{C} 18-\mathrm{H} 18$ & 0.9300 \\
\hline $\mathrm{C} 5-\mathrm{N} 1$ & $1.345(3)$ & $\mathrm{C} 19-\mathrm{C} 20$ & $1.370(5)$ \\
\hline $\mathrm{C} 5-\mathrm{H} 5$ & 0.9300 & $\mathrm{C} 19-\mathrm{H} 19$ & 0.9300 \\
\hline $\mathrm{C} 6-\mathrm{N} 2$ & $1.467(3)$ & $\mathrm{C} 20-\mathrm{C} 21$ & $1.381(4)$ \\
\hline C6-H6A & 0.9700 & $\mathrm{C} 20-\mathrm{H} 20$ & 0.9300 \\
\hline C6-H6B & 0.9700 & $\mathrm{C} 21-\mathrm{H} 21$ & 0.9300 \\
\hline $\mathrm{C} 7-\mathrm{N} 2$ & $1.471(3)$ & $\mathrm{N} 4-\mathrm{H} 4 \mathrm{~A}$ & 0.8600 \\
\hline $\mathrm{C} 7-\mathrm{C} 8$ & $1.516(3)$ & $\mathrm{N} 4-\mathrm{H} 4 \mathrm{~B}$ & 0.8600 \\
\hline $\mathrm{C} 7-\mathrm{H} 7 \mathrm{~A}$ & 0.9700 & $\mathrm{~N} 6-\mathrm{O} 2$ & $1.245(2)$ \\
\hline C7-H7B & 0.9700 & $\mathrm{~N} 6-\mathrm{O} 1$ & $1.246(2)$ \\
\hline $\mathrm{C} 8-\mathrm{H} 8 \mathrm{~A}$ & 0.9600 & $\mathrm{C} 22-\mathrm{C} 23$ & $1.388(8)$ \\
\hline $\mathrm{C} 8-\mathrm{H} 8 \mathrm{~B}$ & 0.9600 & $\mathrm{C} 22-\mathrm{H} 22 \mathrm{~A}$ & 0.9600 \\
\hline $\mathrm{C} 8-\mathrm{H} 8 \mathrm{C}$ & 0.9600 & $\mathrm{C} 22-\mathrm{H} 22 \mathrm{~B}$ & 0.9600 \\
\hline $\mathrm{C} 9-\mathrm{N} 2$ & $1.354(2)$ & $\mathrm{C} 22-\mathrm{H} 22 \mathrm{C}$ & 0.9600 \\
\hline $\mathrm{C} 9-\mathrm{C} 13$ & $1.384(3)$ & $\mathrm{C} 23-\mathrm{O} 3$ & $1.400(9)$ \\
\hline $\mathrm{C} 9-\mathrm{N} 3$ & $1.389(2)$ & $\mathrm{C} 23-\mathrm{H} 23 \mathrm{~A}$ & 0.9700 \\
\hline $\mathrm{C} 10-\mathrm{C} 11$ & $1.348(3)$ & $\mathrm{C} 23-\mathrm{H} 23 \mathrm{~B}$ & 0.9700 \\
\hline $\mathrm{C} 10-\mathrm{N} 4$ & $1.353(3)$ & $\mathrm{O} 3-\mathrm{H} 3 \mathrm{~A}$ & 0.8200 \\
\hline $\mathrm{C} 10-\mathrm{N} 3$ & $1.402(3)$ & $\mathrm{C} 22^{\prime}-\mathrm{C} 23^{\prime}$ & $1.439(8)$ \\
\hline $\mathrm{C} 11-\mathrm{C} 15$ & $1.420(3)$ & $\mathrm{C} 22^{\prime}-\mathrm{H} 22 \mathrm{D}$ & 0.9600 \\
\hline $\mathrm{C} 11-\mathrm{C} 12$ & $1.510(3)$ & $\mathrm{C} 22^{\prime}-\mathrm{H} 22 \mathrm{E}$ & 0.9600 \\
\hline $\mathrm{C} 12-\mathrm{C} 13$ & $1.519(3)$ & $\mathrm{C} 22^{\prime}-\mathrm{H} 22 \mathrm{~F}$ & 0.9600 \\
\hline $\mathrm{C} 12-\mathrm{C} 16$ & $1.523(3)$ & $\mathrm{C} 23^{\prime}-\mathrm{O}^{\prime}$ & $1.370(8)$ \\
\hline $\mathrm{C} 12-\mathrm{H} 12$ & 0.9800 & $\mathrm{C} 23^{\prime}-\mathrm{H} 23 \mathrm{C}$ & 0.9700 \\
\hline $\mathrm{C} 13-\mathrm{N} 6$ & $1.398(3)$ & $\mathrm{C} 23^{\prime}-\mathrm{H} 23 \mathrm{D}$ & 0.9700 \\
\hline $\mathrm{C} 14-\mathrm{N} 3$ & $1.472(3)$ & $\mathrm{O} 3^{\prime}-\mathrm{H} 3 \mathrm{~A}$ & 0.8975 \\
\hline C14-H14A & 0.9600 & $\mathrm{O}^{\prime}{ }^{\prime}-\mathrm{H} 3 \mathrm{~B}$ & 0.8200 \\
\hline $\mathrm{N} 1-\mathrm{C} 1-\mathrm{C} 2$ & $125.0(2)$ & $\mathrm{H} 14 \mathrm{~A}-\mathrm{C} 14-\mathrm{H} 14 \mathrm{C}$ & 109.5 \\
\hline $\mathrm{N} 1-\mathrm{C} 1-\mathrm{Cl1}$ & $115.25(19)$ & $\mathrm{H} 14 \mathrm{~B}-\mathrm{C} 14-\mathrm{H} 14 \mathrm{C}$ & 109.5 \\
\hline $\mathrm{C} 2-\mathrm{C} 1-\mathrm{C} 11$ & $119.72(19)$ & $\mathrm{N} 5-\mathrm{C} 15-\mathrm{C} 11$ & $179.7(3)$ \\
\hline $\mathrm{C} 3-\mathrm{C} 2-\mathrm{C} 1$ & $117.2(2)$ & $\mathrm{C} 21-\mathrm{C} 16-\mathrm{C} 17$ & $117.9(2)$ \\
\hline $\mathrm{C} 3-\mathrm{C} 2-\mathrm{H} 2$ & 121.4 & $\mathrm{C} 21-\mathrm{C} 16-\mathrm{C} 12$ & $119.5(2)$ \\
\hline $\mathrm{C} 1-\mathrm{C} 2-\mathrm{H} 2$ & 121.4 & $\mathrm{C} 17-\mathrm{C} 16-\mathrm{C} 12$ & $122.6(2)$ \\
\hline $\mathrm{C} 2-\mathrm{C} 3-\mathrm{C} 4$ & $120.4(2)$ & $\mathrm{C} 16-\mathrm{C} 17-\mathrm{C} 18$ & $121.0(3)$ \\
\hline $\mathrm{C} 2-\mathrm{C} 3-\mathrm{H} 3$ & 119.8 & $\mathrm{C} 16-\mathrm{C} 17-\mathrm{H} 17$ & 119.5 \\
\hline $\mathrm{C} 4-\mathrm{C} 3-\mathrm{H} 3$ & 119.8 & $\mathrm{C} 18-\mathrm{C} 17-\mathrm{H} 17$ & 119.5 \\
\hline $\mathrm{C} 5-\mathrm{C} 4-\mathrm{C} 3$ & $116.5(2)$ & $\mathrm{C} 19-\mathrm{C} 18-\mathrm{C} 17$ & $120.8(3)$ \\
\hline $\mathrm{C} 5-\mathrm{C} 4-\mathrm{C} 6$ & $120.69(19)$ & $\mathrm{C} 19-\mathrm{C} 18-\mathrm{H} 18$ & 119.6 \\
\hline $\mathrm{C} 3-\mathrm{C} 4-\mathrm{C} 6$ & $122.7(2)$ & $\mathrm{C} 17-\mathrm{C} 18-\mathrm{H} 18$ & 119.6 \\
\hline $\mathrm{N} 1-\mathrm{C} 5-\mathrm{C} 4$ & $124.6(2)$ & $\mathrm{C} 18-\mathrm{C} 19-\mathrm{C} 20$ & $119.0(3)$ \\
\hline $\mathrm{N} 1-\mathrm{C} 5-\mathrm{H} 5$ & 117.7 & $\mathrm{C} 18-\mathrm{C} 19-\mathrm{H} 19$ & 120.5 \\
\hline $\mathrm{C} 4-\mathrm{C} 5-\mathrm{H} 5$ & 117.7 & $\mathrm{C} 20-\mathrm{C} 19-\mathrm{H} 19$ & 120.5 \\
\hline $\mathrm{N} 2-\mathrm{C} 6-\mathrm{C} 4$ & $113.92(16)$ & $\mathrm{C} 19-\mathrm{C} 20-\mathrm{C} 21$ & $120.6(3)$ \\
\hline $\mathrm{N} 2-\mathrm{C} 6-\mathrm{H} 6 \mathrm{~A}$ & 108.8 & $\mathrm{C} 19-\mathrm{C} 20-\mathrm{H} 20$ & 119.7 \\
\hline $\mathrm{C} 4-\mathrm{C} 6-\mathrm{H} 6 \mathrm{~A}$ & 108.8 & $\mathrm{C} 21-\mathrm{C} 20-\mathrm{H} 20$ & 119.7 \\
\hline
\end{tabular}




\begin{tabular}{|c|c|c|c|}
\hline $\mathrm{N} 2-\mathrm{C} 6-\mathrm{H} 6 \mathrm{~B}$ & 108.8 & $\mathrm{C} 16-\mathrm{C} 21-\mathrm{C} 20$ & $120.7(3)$ \\
\hline $\mathrm{C} 4-\mathrm{C} 6-\mathrm{H} 6 \mathrm{~B}$ & 108.8 & $\mathrm{C} 16-\mathrm{C} 21-\mathrm{H} 21$ & 119.6 \\
\hline $\mathrm{H} 6 \mathrm{~A}-\mathrm{C} 6-\mathrm{H} 6 \mathrm{~B}$ & 107.7 & $\mathrm{C} 20-\mathrm{C} 21-\mathrm{H} 21$ & 119.6 \\
\hline $\mathrm{N} 2-\mathrm{C} 7-\mathrm{C} 8$ & $112.2(2)$ & $\mathrm{C} 1-\mathrm{N} 1-\mathrm{C} 5$ & $116.1(2)$ \\
\hline $\mathrm{N} 2-\mathrm{C} 7-\mathrm{H} 7 \mathrm{~A}$ & 109.2 & $\mathrm{C} 9-\mathrm{N} 2-\mathrm{C} 6$ & $122.86(17)$ \\
\hline $\mathrm{C} 8-\mathrm{C} 7-\mathrm{H} 7 \mathrm{~A}$ & 109.2 & $\mathrm{C} 9-\mathrm{N} 2-\mathrm{C} 7$ & $120.18(18)$ \\
\hline $\mathrm{N} 2-\mathrm{C} 7-\mathrm{H} 7 \mathrm{~B}$ & 109.2 & $\mathrm{C} 6-\mathrm{N} 2-\mathrm{C} 7$ & $115.36(15)$ \\
\hline $\mathrm{C} 8-\mathrm{C} 7-\mathrm{H} 7 \mathrm{~B}$ & 109.2 & $\mathrm{C} 9-\mathrm{N} 3-\mathrm{C} 10$ & $119.57(16)$ \\
\hline $\mathrm{H} 7 \mathrm{~A}-\mathrm{C} 7-\mathrm{H} 7 \mathrm{~B}$ & 107.9 & $\mathrm{C} 9-\mathrm{N} 3-\mathrm{C} 14$ & $121.28(16)$ \\
\hline $\mathrm{C} 7-\mathrm{C} 8-\mathrm{H} 8 \mathrm{~A}$ & 109.5 & $\mathrm{C} 10-\mathrm{N} 3-\mathrm{C} 14$ & $118.32(17)$ \\
\hline $\mathrm{C} 7-\mathrm{C} 8-\mathrm{H} 8 \mathrm{~B}$ & 109.5 & $\mathrm{C} 10-\mathrm{N} 4-\mathrm{H} 4 \mathrm{~A}$ & 120.0 \\
\hline $\mathrm{H} 8 \mathrm{~A}-\mathrm{C} 8-\mathrm{H} 8 \mathrm{~B}$ & 109.5 & $\mathrm{C} 10-\mathrm{N} 4-\mathrm{H} 4 \mathrm{~B}$ & 120.0 \\
\hline $\mathrm{C} 7-\mathrm{C} 8-\mathrm{H} 8 \mathrm{C}$ & 109.5 & $\mathrm{H} 4 \mathrm{~A}-\mathrm{N} 4-\mathrm{H} 4 \mathrm{~B}$ & 120.0 \\
\hline $\mathrm{H} 8 \mathrm{~A}-\mathrm{C} 8-\mathrm{H} 8 \mathrm{C}$ & 109.5 & $\mathrm{O} 2-\mathrm{N} 6-\mathrm{O} 1$ & $120.54(17)$ \\
\hline $\mathrm{H} 8 \mathrm{~B}-\mathrm{C} 8-\mathrm{H} 8 \mathrm{C}$ & 109.5 & $\mathrm{O} 2-\mathrm{N} 6-\mathrm{C} 13$ & $121.09(18)$ \\
\hline $\mathrm{N} 2-\mathrm{C} 9-\mathrm{C} 13$ & $128.39(18)$ & $\mathrm{O} 1-\mathrm{N} 6-\mathrm{C} 13$ & $118.36(17)$ \\
\hline $\mathrm{N} 2-\mathrm{C} 9-\mathrm{N} 3$ & $114.80(17)$ & $\mathrm{C} 22-\mathrm{C} 23-\mathrm{O} 3$ & $119.7(11)$ \\
\hline $\mathrm{C} 13-\mathrm{C} 9-\mathrm{N} 3$ & $116.72(17)$ & $\mathrm{C} 22-\mathrm{C} 23-\mathrm{H} 23 \mathrm{~A}$ & 107.4 \\
\hline $\mathrm{C} 11-\mathrm{C} 10-\mathrm{N} 4$ & $126.1(2)$ & $\mathrm{O} 3-\mathrm{C} 23-\mathrm{H} 23 \mathrm{~A}$ & 107.4 \\
\hline $\mathrm{C} 11-\mathrm{C} 10-\mathrm{N} 3$ & $119.12(19)$ & $\mathrm{C} 22-\mathrm{C} 23-\mathrm{H} 23 \mathrm{~B}$ & 107.4 \\
\hline $\mathrm{N} 4-\mathrm{C} 10-\mathrm{N} 3$ & $114.74(19)$ & $\mathrm{O} 3-\mathrm{C} 23-\mathrm{H} 23 \mathrm{~B}$ & 107.4 \\
\hline $\mathrm{C} 10-\mathrm{C} 11-\mathrm{C} 15$ & $119.8(2)$ & $\mathrm{H} 23 \mathrm{~A}-\mathrm{C} 23-\mathrm{H} 23 \mathrm{~B}$ & 106.9 \\
\hline $\mathrm{C} 10-\mathrm{C} 11-\mathrm{C} 12$ & $119.17(18)$ & $\mathrm{C} 23-\mathrm{O} 3-\mathrm{H} 3 \mathrm{~A}$ & 100.2 \\
\hline $\mathrm{C} 15-\mathrm{C} 11-\mathrm{C} 12$ & $120.9(2)$ & $\mathrm{C} 23^{\prime}-\mathrm{C} 22^{\prime}-\mathrm{H} 22 \mathrm{D}$ & 109.5 \\
\hline $\mathrm{C} 11-\mathrm{C} 12-\mathrm{C} 13$ & $105.76(17)$ & $\mathrm{C} 23^{\prime}-\mathrm{C} 22^{\prime}-\mathrm{H} 22 \mathrm{E}$ & 109.5 \\
\hline $\mathrm{C} 11-\mathrm{C} 12-\mathrm{C} 16$ & $114.75(17)$ & $\mathrm{H} 22 \mathrm{D}-\mathrm{C} 22^{\prime}-\mathrm{H} 22 \mathrm{E}$ & 109.5 \\
\hline $\mathrm{C} 13-\mathrm{C} 12-\mathrm{C} 16$ & $112.45(17)$ & $\mathrm{C} 23^{\prime}-\mathrm{C} 22^{\prime}-\mathrm{H} 22 \mathrm{~F}$ & 109.5 \\
\hline $\mathrm{C} 11-\mathrm{C} 12-\mathrm{H} 12$ & 107.9 & $\mathrm{H} 22 \mathrm{D}-\mathrm{C} 22^{\prime}-\mathrm{H} 22 \mathrm{~F}$ & 109.5 \\
\hline $\mathrm{C} 13-\mathrm{C} 12-\mathrm{H} 12$ & 107.9 & $\mathrm{H} 22 \mathrm{E}-\mathrm{C} 22^{\prime}-\mathrm{H} 22 \mathrm{~F}$ & 109.5 \\
\hline $\mathrm{C} 16-\mathrm{C} 12-\mathrm{H} 12$ & 107.9 & $\mathrm{O} 3^{\prime}-\mathrm{C} 23^{\prime}-\mathrm{C} 22^{\prime}$ & $111.9(11)$ \\
\hline $\mathrm{C} 9-\mathrm{C} 13-\mathrm{N} 6$ & $122.37(17)$ & $\mathrm{O} 3^{\prime}-\mathrm{C} 23^{\prime}-\mathrm{H} 23 \mathrm{C}$ & 109.2 \\
\hline $\mathrm{C} 9-\mathrm{C} 13-\mathrm{C} 12$ & $119.35(17)$ & $\mathrm{C} 22^{\prime}-\mathrm{C} 23^{\prime}-\mathrm{H} 23 \mathrm{C}$ & 109.2 \\
\hline $\mathrm{N} 6-\mathrm{C} 13-\mathrm{C} 12$ & $117.72(17)$ & $\mathrm{O} 3^{\prime}-\mathrm{C} 23^{\prime}-\mathrm{H} 23 \mathrm{D}$ & 109.2 \\
\hline N3-C14-H14A & 109.5 & $\mathrm{C} 22^{\prime}-\mathrm{C} 23^{\prime}-\mathrm{H} 23 \mathrm{D}$ & 109.2 \\
\hline N3-C14-H14B & 109.5 & $\mathrm{H} 23 \mathrm{C}-\mathrm{C} 23^{\prime}-\mathrm{H} 23 \mathrm{D}$ & 107.9 \\
\hline $\mathrm{H} 14 \mathrm{~A}-\mathrm{C} 14-\mathrm{H} 14 \mathrm{~B}$ & 109.5 & $\mathrm{C} 23^{\prime}-\mathrm{O} 3^{\prime}-\mathrm{H} 3 \mathrm{~A}$ & 107.5 \\
\hline $\mathrm{N} 3-\mathrm{C} 14-\mathrm{H} 14 \mathrm{C}$ & 109.5 & $\mathrm{C} 23^{\prime}-\mathrm{O}^{\prime}-\mathrm{H} 3 \mathrm{~B}$ & 109.5 \\
\hline $\mathrm{N} 1-\mathrm{C} 1-\mathrm{C} 2-\mathrm{C} 3$ & $2.3(4)$ & $\mathrm{C} 12-\mathrm{C} 16-\mathrm{C} 17-\mathrm{C} 18$ & $178.2(2)$ \\
\hline $\mathrm{C} 11-\mathrm{C} 1-\mathrm{C} 2-\mathrm{C} 3$ & $-177.31(18)$ & $\mathrm{C} 16-\mathrm{C} 17-\mathrm{C} 18-\mathrm{C} 19$ & $0.7(4)$ \\
\hline $\mathrm{C} 1-\mathrm{C} 2-\mathrm{C} 3-\mathrm{C} 4$ & $-0.4(3)$ & $\mathrm{C} 17-\mathrm{C} 18-\mathrm{C} 19-\mathrm{C} 20$ & $-0.2(5)$ \\
\hline $\mathrm{C} 2-\mathrm{C} 3-\mathrm{C} 4-\mathrm{C} 5$ & $-1.5(3)$ & $\mathrm{C} 18-\mathrm{C} 19-\mathrm{C} 20-\mathrm{C} 21$ & $0.1(5)$ \\
\hline $\mathrm{C} 2-\mathrm{C} 3-\mathrm{C} 4-\mathrm{C} 6$ & $174.9(2)$ & $\mathrm{C} 17-\mathrm{C} 16-\mathrm{C} 21-\mathrm{C} 20$ & $0.8(4)$ \\
\hline $\mathrm{C} 3-\mathrm{C} 4-\mathrm{C} 5-\mathrm{N} 1$ & $1.9(3)$ & $\mathrm{C} 12-\mathrm{C} 16-\mathrm{C} 21-\mathrm{C} 20$ & $-178.4(2)$ \\
\hline $\mathrm{C} 6-\mathrm{C} 4-\mathrm{C} 5-\mathrm{N} 1$ & $-174.6(2)$ & $\mathrm{C} 19-\mathrm{C} 20-\mathrm{C} 21-\mathrm{C} 16$ & $-0.4(4)$ \\
\hline $\mathrm{C} 5-\mathrm{C} 4-\mathrm{C} 6-\mathrm{N} 2$ & $-127.6(2)$ & $\mathrm{C} 2-\mathrm{C} 1-\mathrm{N} 1-\mathrm{C} 5$ & $-2.1(4)$ \\
\hline $\mathrm{C} 3-\mathrm{C} 4-\mathrm{C} 6-\mathrm{N} 2$ & $56.2(3)$ & $\mathrm{C} 11-\mathrm{C} 1-\mathrm{N} 1-\mathrm{C} 5$ & $177.59(17)$ \\
\hline $\mathrm{N} 4-\mathrm{C} 10-\mathrm{C} 11-\mathrm{C} 15$ & $-8.0(3)$ & $\mathrm{C} 4-\mathrm{C} 5-\mathrm{N} 1-\mathrm{C} 1$ & $-0.1(4)$ \\
\hline
\end{tabular}




$\begin{array}{llll}\mathrm{N} 3-\mathrm{C} 10-\mathrm{C} 11-\mathrm{C} 15 & 175.28(19) & \mathrm{C} 13-\mathrm{C} 9-\mathrm{N} 2-\mathrm{C} 6 & 31.1(3) \\ \mathrm{N} 4-\mathrm{C} 10-\mathrm{C} 11-\mathrm{C} 12 & 168.5(2) & \mathrm{N} 3-\mathrm{C} 9-\mathrm{N} 2-\mathrm{C} 6 & -145.33(18) \\ \mathrm{N} 3-\mathrm{C} 10-\mathrm{C} 11-\mathrm{C} 12 & -8.2(3) & \mathrm{C} 13-\mathrm{C} 9-\mathrm{N} 2-\mathrm{C} 7 & -133.8(2) \\ \mathrm{C} 10-\mathrm{C} 11-\mathrm{C} 12-\mathrm{C} 13 & 40.0(2) & \mathrm{N} 3-\mathrm{C} 9-\mathrm{N} 2-\mathrm{C} 7 & 49.8(2) \\ \mathrm{C} 15-\mathrm{C} 11-\mathrm{C} 12-\mathrm{C} 13 & -143.46(19) & \mathrm{C} 4-\mathrm{C} 6-\mathrm{N} 2-\mathrm{C} 9 & 40.7(3) \\ \mathrm{C} 10-\mathrm{C} 11-\mathrm{C} 12-\mathrm{C} 16 & -84.5(2) & \mathrm{C} 4-\mathrm{C} 6-\mathrm{N} 2-\mathrm{C} 7 & -153.71(17) \\ \mathrm{C} 15-\mathrm{C} 11-\mathrm{C} 12-\mathrm{C} 16 & 92.0(2) & \mathrm{C} 8-\mathrm{C} 7-\mathrm{N} 2-\mathrm{C} 9 & 101.8(2) \\ \mathrm{N} 2-\mathrm{C} 9-\mathrm{C} 13-\mathrm{N} 6 & 27.3(3) & \mathrm{C} 8-\mathrm{C} 7-\mathrm{N} 2-\mathrm{C} 6 & -64.2(2) \\ \mathrm{N} 3-\mathrm{C} 9-\mathrm{C} 13-\mathrm{N} 6 & -156.32(18) & \mathrm{N} 2-\mathrm{C} 9-\mathrm{N} 3-\mathrm{C} 10 & -160.22(17) \\ \mathrm{N} 2-\mathrm{C} 9-\mathrm{C} 13-\mathrm{C} 12 & -161.53(19) & \mathrm{C} 13-\mathrm{C} 9-\mathrm{N} 3-\mathrm{C} 10 & 22.9(3) \\ \mathrm{N} 3-\mathrm{C} 9-\mathrm{C} 13-\mathrm{C} 12 & 14.9(3) & \mathrm{N} 2-\mathrm{C} 9-\mathrm{N} 3-\mathrm{C} 14 & 30.4(3) \\ \mathrm{C} 11-\mathrm{C} 12-\mathrm{C} 13-\mathrm{C} 9 & -43.8(2) & \mathrm{C} 13-\mathrm{C} 9-\mathrm{N} 3-\mathrm{C} 14 & -146.51(19) \\ \mathrm{C} 16-\mathrm{C} 12-\mathrm{C} 13-\mathrm{C} 9 & 82.2(2) & \mathrm{C} 11-\mathrm{C} 10-\mathrm{N} 3-\mathrm{C} 9 & -26.8(3) \\ \mathrm{C} 11-\mathrm{C} 12-\mathrm{C} 13-\mathrm{N} 6 & 127.84(19) & \mathrm{N} 4-\mathrm{C} 10-\mathrm{N} 3-\mathrm{C} 9 & 156.08(19) \\ \mathrm{C} 16-\mathrm{C} 12-\mathrm{C} 13-\mathrm{N} 6 & -106.2(2) & \mathrm{C} 11-\mathrm{C} 10-\mathrm{N} 3-\mathrm{C} 14 & 142.9(2) \\ \mathrm{C} 11-\mathrm{C} 12-\mathrm{C} 16-\mathrm{C} 21 & -164.9(2) & \mathrm{N} 4-\mathrm{C} 10-\mathrm{N} 3-\mathrm{C} 14 & -34.2(3) \\ \mathrm{C} 13-\mathrm{C} 12-\mathrm{C} 16-\mathrm{C} 21 & 74.1(3) & \mathrm{C} 9-\mathrm{C} 13-\mathrm{N} 6-\mathrm{O} 2 & 6.9(3) \\ \mathrm{C} 11-\mathrm{C} 12-\mathrm{C} 16-\mathrm{C} 17 & 15.9(3) & \mathrm{C} 12-\mathrm{C} 13-\mathrm{N} 6-\mathrm{O} 2 & -164.43(18) \\ \mathrm{C} 13-\mathrm{C} 12-\mathrm{C} 16-\mathrm{C} 17 & -105.0(2) & \mathrm{C} 9-\mathrm{C} 13-\mathrm{N} 6-\mathrm{O} 1 & -174.0(2) \\ \mathrm{C} 21-\mathrm{C} 16-\mathrm{C} 17-\mathrm{C} 18 & -1.0(4) & \mathrm{C} 12-\mathrm{C} 13-\mathrm{N} 6-\mathrm{O} 1 & 14.6(3) \\ \end{array}$

Hydrogen-bond geometry $\left(A,{ }^{\circ}\right)$

\begin{tabular}{lllll}
\hline$D-\mathrm{H} \cdots A$ & $D-\mathrm{H}$ & $\mathrm{H} \cdots A$ & $D \cdots A$ & $D-\mathrm{H} \cdots A$ \\
\hline $\mathrm{O} 3-\mathrm{H} 3 A \cdots \mathrm{N} 1^{\mathrm{i}}$ & 0.82 & 1.97 & $2.785(13)$ & 179 \\
$\mathrm{~N} 4-\mathrm{H} 4 B \cdots \mathrm{O} 3$ & 0.86 & 2.26 & $2.902(11)$ & 132 \\
$\mathrm{C} 6-\mathrm{H} 6 A \cdots \mathrm{O} 2$ & 0.97 & 2.13 & $2.783(3)$ & 124 \\
$\mathrm{C} 7-\mathrm{H} 7 B \cdots \mathrm{O} 22^{\mathrm{ii}}$ & 0.97 & 2.57 & $3.378(3)$ & 141 \\
\hline
\end{tabular}

Symmetry codes: (i) $x,-y+1 / 2, z-1 / 2$; (ii) $-x+1 / 2, y-1 / 2, z$. 